\title{
Investigation on Heavy Precipitation Effects over FSO Link
}

\author{
M.F. Talib ${ }^{1, *}$, A.K. $\operatorname{Rahman}^{1}$, M.S. Anuar ${ }^{1}$, C.B.M. Rashidi ${ }^{1}$, and S.A. Aljunid ${ }^{1}$ \\ ${ }^{1}$ Advance Communication Engineering, Centre of Excellence (ACE-CoE), \\ School of Computer \& Communication Engineering University Malaysia Perlis (UniMAP)
}

\begin{abstract}
This paper has examined the performance of free space optic system and scattering effects over the laser propagation. Analysis result based on Optisystem simulation software and rain data collected from Malaysia Meteorological Department Perlis, which covering beneath the tropical rainforest weather. The measurement in a simulation using the real parameter from Laserbit system which using wavelength $785 \mathrm{~nm}, 0.5-$ $15 \mathrm{mrad}$ beam divergence, $70 \mathrm{~mW}$ laser power and range between $100 \mathrm{~m}-$ $2500 \mathrm{~m}$. The result can estimate the intensity of rain to impair the link and alternative method to overcome the drawback system.
\end{abstract}

\section{Introduction}

In telecommunications, Free Space Optics (FSO) are an optical communication technology that uses light propagating in free space to transmit data between two points. This technology is new in Malaysia and not widely use. It might relate to environmental issues that familiar with this system. FSO also well known as "free space photonic" or "optical wireless" which is classified as unguided beam. Various applications can be deployed in FSO system such as Bridging Wireless Local Area Network (WLAN), LAN to LAN Building Connectivity, Education Campus Network, Gigabit Ethernet, Access and Backhaul. Among the advantage of FSO is not required license from Federal Communication Commission (FCC) for installation. The latest technology in FSO which is design to deploy behind window has eliminating the need for costly rooftops rights. In terms of communication security, FSO provides far more secure than other wireless-based band transmission, such as uses narrow laser beam light making harder to intercept and crack [1].

Unfortunately, FSO system is vulnerable to atmosphere phenomenon such as attenuation and scintillation which can reduce laser power and limit the path length for a given link availability. Basically, in FSO the impairments link is caused by air turbulence that produces a beam spreading, beam wander, scintillation and degradation of the coherence of the wave front [2] and hydrometers (rain and snow) and suspended water particles (fog represents the worst meteorological situation for FSO) that yield high losses due to scattering $[3,7,8]$.

\footnotetext{
* Corresponding author: karahman@unimas.my
} 


\section{Theoretical approaches}

\subsection{Scattering coefficient}

Rain is liquid precipitation. It is the condensation of atmospheric water vapour into drops heavy enough to fall, often making it to the surface and the most frequent type of atmospheric precipitation in temperate climates. Rain is under non-selective or geometrical scattering where it occurs in the lower portion of the atmosphere when the particles are much larger than the incident radiation. The scattering coefficient can be calculated by using Stroke Law [3].

$$
\beta_{\text {rainscat }}=\pi a^{2} N_{a} Q_{\text {scat }}\left(\frac{a}{\lambda}\right)
$$

Where:

$a \quad=$ radius of raindrop $(0.001 \mathrm{~cm}$ to $0.1 \mathrm{~cm})$

$N_{a} \quad=$ Rain drop distribution

$Q_{\text {scat }}=$ scattering efficiency

$\lambda \quad=$ wavelength

\subsection{Atmospheric attenuation}

The attenuation of laser power in the atmosphere is described by Beer's Law [5]:

$$
\tau(R)=-\frac{P(R)}{P(O)}=e^{-(\beta) R}
$$

$\tau(R)=$ transmittance at range $\mathrm{R}$

$P(R)=$ laser power at $\mathrm{R}$

$P(O)=$ laser power at the source

$\beta \quad=$ scattering coefficient

$R \quad=$ link range $(\mathrm{km})$

Equation (2) can be converted to logarithms scale using Equation (3)

$$
\begin{aligned}
& =-10 \log \frac{P(R)}{P(O)} \\
& =-10 \log e^{-\beta R} \\
& =10 \log e^{-\beta R}
\end{aligned}
$$

\section{Result \& discussion}

Fig 1 shows the performance of the scattering coefficient against intensity rain. The $\mathrm{x}$-axis represents the intense rain varies from $0-0.00045 \mathrm{~cm} / \mathrm{sec}$ and $\mathrm{y}$-axis represent the scattering coefficient in $\mathrm{km}^{-1}$. The curves were plotted based on Equation (1). 


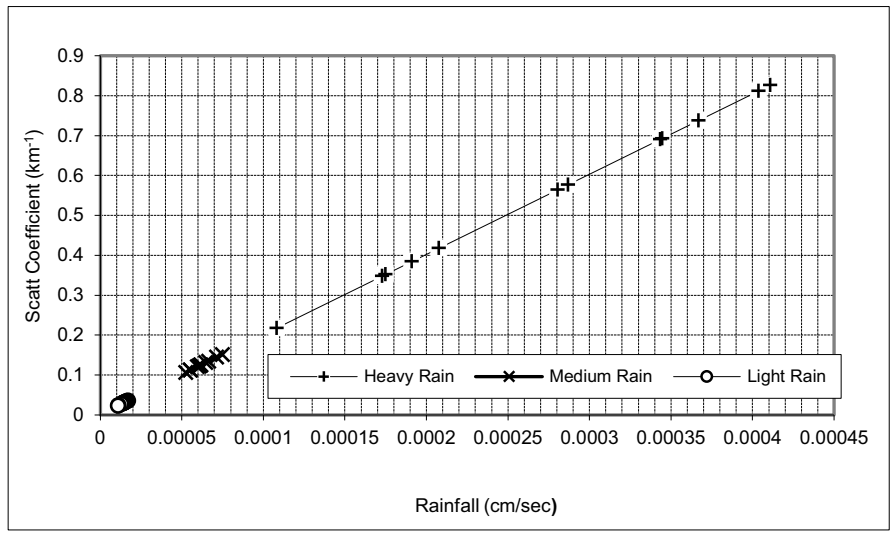

Fig. 1. Scattering Coefficient against Rainfall rate.

The graph also indicates the performance of three different rain i.e. The heavy rain, medium rain and light rain. Scattering for light rain varies from $0.022 \mathrm{~km}^{-1}$ to $0.035 \mathrm{~km}^{-1}$ while for medium rain varies from $0.112 \mathrm{~km}^{-1}$ to $0.151 \mathrm{~km}^{-1}$ and for heavy rain varies from $0.218 \mathrm{~km}^{-1}$ to $0.827 \mathrm{~km}^{-1}$.

From the analysis the heavy rain exhibits a high scattering coefficient effect comparable to the other rain type. The heavy rain rate varies from $0.0001 \mathrm{~cm} / \mathrm{sec}$ to $0.00045 \mathrm{~cm} / \mathrm{sec}$. The graph also shows that the scattering coefficient is linearly proportional with rainfall intensity. The highest precipitation rate will produce a high scattering coefficient. In this context scattering coefficient mean a measure of the light attenuation due to scattering of light as it traverses a medium containing scattering particles. Thus the rise of scattering coefficient will yield high atmospheric attenuation with respect of precipitation rate and this relationship will be elaborate more in Fig 2.

The $\mathrm{x}$-axis represents rainfall rate and $\mathrm{y}$-axis correspond to atmospheric attenuation. Fig 2 proves that the atmospheric attenuation is linearly proportional with rainfall rate. The curve obtained from Equation (2) from Beer's Law [4] which explain that laser power at receiver over laser power at transmittance is equivalent with exponential scattering coefficient times link range of laser transmission. Link range between transmitter and receiver is within 0.5 kilometer. As the rainfall rate increases, the attenuation will increase as well.

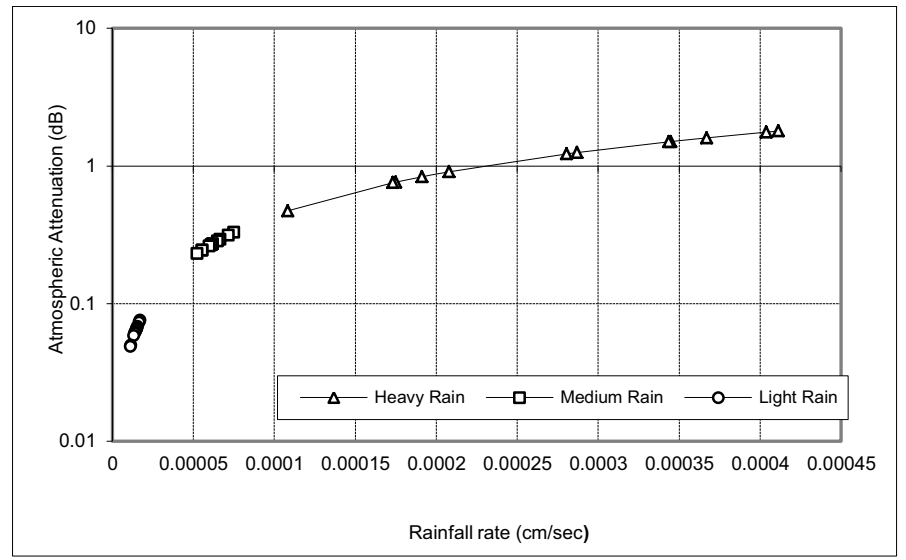

Fig. 2. Atmospheric Attenuation due to intensity rain. 
Basically, the graph shows three different types of rain. The high atmospheric attenuation is measure at $1.7949 \mathrm{~dB}$ and lowest attenuation at $0.0485 \mathrm{~dB}$. Mostly severe rain (heavy rain) yields $0.4730 \mathrm{~dB}-1.7939 \mathrm{~dB}$ and medium rain and light rain below $1 \mathrm{~dB}$ changing from $0.3274 \mathrm{~dB}-0.2292 \mathrm{~dB}$ and $0.0728 \mathrm{~dB}-0.0485 \mathrm{~dB}$ respectively. As explain in Fig 1 the high scattering contributes high atmospheric attenuation. However, these attenuation not enough to impair the link but as the link range increases the atmospheric attenuation begin to increase. All of the can observe in Fig 3 which show the relation laser power at receiver with link distance.

Fig 3 shows the performance of the heavy rain due to link distance. The powers at receiver laser in range $20 \mathrm{dBm}$ to $-120 \mathrm{dBm}$ and distance about $5 \mathrm{~km}$. The curve is obtained by simulation using Optisystem software. The equation that use to simulate this curve is

$$
P_{\text {received }}=P_{\text {transmit }} \frac{d_{R}{ }^{2} R}{\left(d_{T}+\theta R\right)^{2}} \times 10^{-\left(\frac{\alpha R}{10}\right)}
$$

$d_{R}=$ Receiver Aperture $(\mathrm{cm})$

$d_{T}=$ Transmitter Aperture $(\mathrm{cm})$

$\theta=$ Beam divergence (mrad)

$\alpha=$ Atmospheric attenuation $(\mathrm{dB} / \mathrm{km})$

From the equation, the input parameter is based on laser bit equipment. Receiver aperture $=$ $20 \mathrm{~cm}$, transmitter aperture $=5 \mathrm{~cm}$, beam divergence $=8 \mathrm{mrad}$, wavelength $=785 \mathrm{~nm}$ and power consumed $=70 \mathrm{~mW}$.

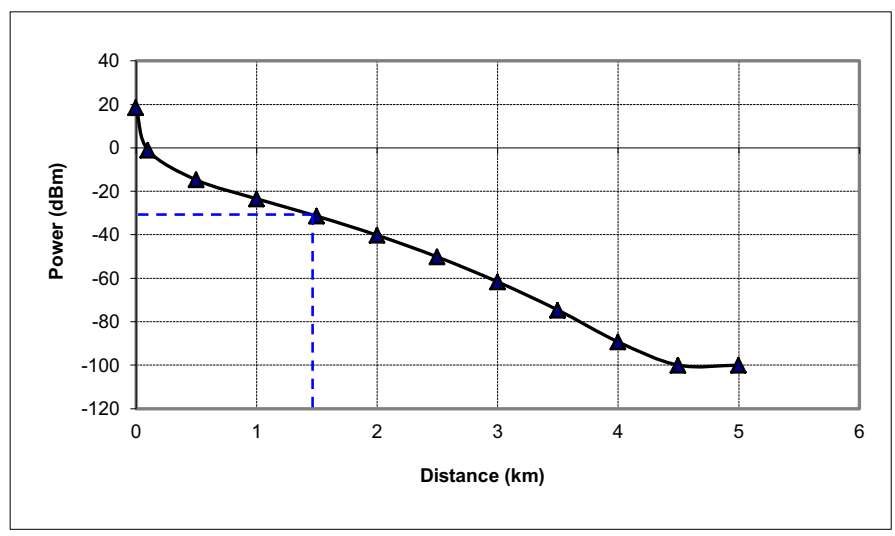

Fig. 3. The changing laser power at receiver due to increasing link distance.

The graph shows that power laser is inversely proportional with link distance. As the distance increases, the power at receiver decreases. This condition depict that when power laser transmit through heavy rain it experiences weaken power. The blue dot lines indicate the minimum sufficient laser power at receiver for full modulation transmission about $31.438 \mathrm{dBm}$ with range $1.5 \mathrm{~km}$. When power continue to decreases, the link begin to impair and can drop it worst condition totally error. The minimum sufficient laser power is vital because represent the link performance under heavy rain but if the receiver receives below minimum power big possibility data error in transmission is high. This result based on Optisystem simulation in Bit Error Rate (BER) Analyser that has been done.

The solution for this problem is to setup short range between transmitter and receiver. As we can see in Fig 3 above, the distance between 0 less than $1.5 \mathrm{~km}$ is best range and 
above minimum power require at receiver. Thus can optimize line transmission without add any cost to upgrade the link performance.

Another alternative solution is to use narrow laser beam. This can be implementing by using small beam divergence. Previous parameter use beam divergence $=8 \mathrm{mrad}$. Fig 4 represents power laser correspond with link distance by using beam divergence $=1 \mathrm{mrad}$.

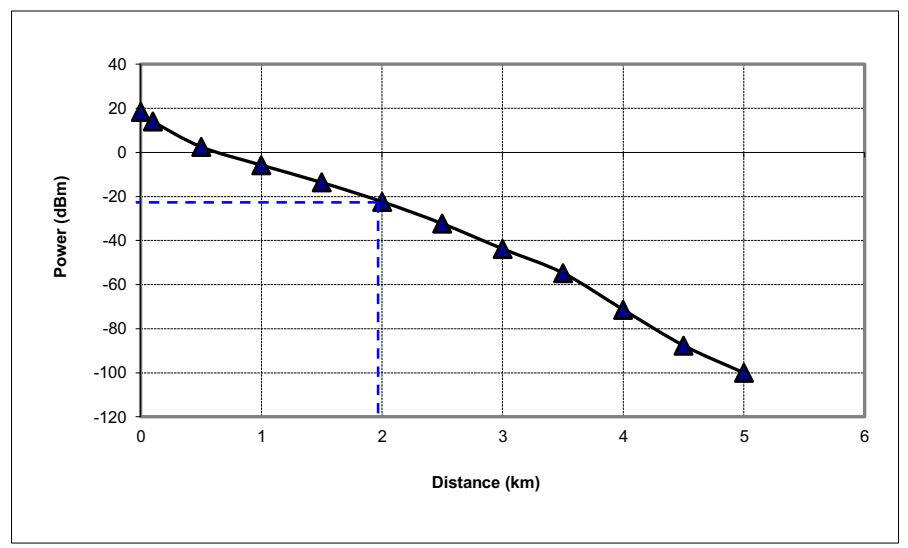

Fig. 4. Relation performance of laser power with link distance (using divergence $=1 \mathrm{mrad}$ ).

Based on the graph, laser powers receive at receiver in scale 20 to $-100 \mathrm{dBm}$ and minimum sufficient power at receiver is $-22.341 \mathrm{dBm}$ at range $2 \mathrm{~km}$. Compare to beam divergence a $8 \mathrm{mrad}$, the minimum sufficient power at range $1.5 \mathrm{~km}$ with distance different about $0.5 \mathrm{~km}$. In laser communication, the more capable system to go further is better because can reduce the usage of laser at short range and moreover add wide coverage communication.

The beam divergence usually provides by vendors. Consumption of wide beam is to make sure enough beam receive at detector and it is to ensure automatically stay align even if small shifting point happens. However, this scheme is acceptable for low data rate only. The advantage of narrow beam can generate high data rates plus add more distance to transmit.

The relationship between beam divergences with distance is illustrated in equation (5) below [5]:

$$
W=R \theta
$$

$W=$ width (or diameter beam area)

$R=$ link distance

$\theta=$ beam divergences

Therefore to avoid larger spread beam it better to use narrow beam such $1 \mathrm{mrad}[6,7]$.

\section{Conclusions}

Rain is the main major effect in the design of FSO system operating in high rainfall regions. The usage of laserbit at $785 \mathrm{~nm}$ wavelength can optimize the line transmission with setup short distance between transmitter and receiver. It can reduce the atmospheric attenuation and fulfil the minimum required laser power at transmitter for full modulation at range below $1.5 \mathrm{~km}$. Other than that the use of narrow beam is suitable in high rainfall region because produce high power at receiver for better transmission. It also gives the 
advantage in data rates transfer. Since FSO bandwidth can up into gigabit it can carry a tremendous amount of information and narrow beam is suitable for high data rate transfer. Therefore a small beam divergence is much better compared to big beam divergence.

This work has been supported by a grant from the Fundamental Research Grant Scheme (FRGS) in FSO research area under ACE - CoE School Communication and Computer, University Malaysia Perlis.

\section{References}

1. M. Achour, Proceedings of SPIE, (2002)

2. D. Deirmendjian, J. Appl. Meteorol., 14, 15584 (1975)

3. J.P.V Poiares Baptista, Precipitation, Clouds and other relate non- refractive effect, (ESA Publication Division, Netherlands)

4. H.Weichel, Laser beam Propagation in the Atmosphere (SPIE Press, Bellingham, 1990)

5. Pedrotti, L. Frank, S. Pedrotti Leno, Introduction To Optics, $2^{\text {nd }}$ edition, (Prentice Hall International Editions, USA, 1993)

6. A.K. Rahman, M.S. Anuar, S.A. Aljunid, M.N. Ismail, B.S. Naimullah, International Conference on Electronic Design, (2008)

7. A.K. Rahman, M.S. Anuar, S.A. Aljunid, M.N. Junita, Proceedings of IEEE $20086^{\text {th }}$ National Conference on Telecommunication Technologies, (2008) 